\title{
Développer les compétences émotionnelles des futur.e.s enseignant.e.s en stage et diminuer leur stress perçu grâce à un programme d'intervention : une étude exploratoire
}

Auteurs

René Langevin, Professeur, Université d'Alberta, Canada, rene.langevin@ualberta.ca

Angélique Laurent, Professeure, Université de Sherbrooke, Canada, Angelique.Laurent@usherbrooke.ca 


\section{REVUE HYBRIDE DE L'ÉDUCATION}

\section{Résumé}

L'objectif de cette étude exploratoire est d'observer les effets d'un programme portant sur les compétences émotionnelles (CE) offert à de futur.e.s enseignant.e.s durant leur stage, l'amélioration de leurs CE et de leur niveau de stress perçu. Les cinq composantes de base des CE et le stress perçu ont été évalués avant et après l'intervention. Malgré un nombre restreint de participant.e.s, les résultats suggèrent une diminution du niveau de stress et une augmentation de la plupart des CE des participant.e.s du groupe expérimental en cours de stage. Ces résultats appuient ceux mis en lumière dans certaines études antérieures qui traitent de questions similaires.

Mots-clés : futur.e.s enseignant.e.s ; stages ; compétences émotionnelles (CE) ; stress perçu ; programme d'intervention 


\section{8}

\section{REVUE HYBRIDE DE L'ÉDUCATION}

\section{Problématique}

Pour les futur.e.s enseignant.e.s, les stages constituent une expérience marquante qui comporte son lot de satisfactions et de contraintes (Vandercleyen et al., 2019). En effet, même si les stages sont sources de plaisir, ils peuvent aussi s'avérer stressants pour certains, voire anxiogène pour d'autres (Pelletier, 2015). Pour plusieurs stagiaires, l'établissement de relations harmonieuses et significatives avec les élèves, l'enseignant.e accompagnateur.rice et la personne superviseur de stage de l'université, représente un enjeu majeur (Desbiens, 2012). Ce genre d'enjeu peut causer du stress, d'autant plus lorsque le stagiaire vit des relations difficiles avec des personnes de son entourage (Bonello et al., 2019). Nous n'avons qu'à penser à une relation conflictuelle entre un stagiaire et son enseignant.e accompagnateur.rice (Weber, 2015).

En ce qui a trait au domaine de l'éducation spécifiquement, rares sont les universités canadiennes ou à l'échelle internationale qui offrent des programmes sur le développement des CE aux futur.e.s enseignant.e.s en formation initiale (Beaumont et Garcia, 2020). Pourtant, ce type de compétence s'avère essentiel pour ces futur.e.s professionnel.le.s puisque leur profession repose notamment sur la qualité des relations interpersonnelles (Goyette et al., 2020). De ce fait, chaque situation professionnelle vécue entraîne, sans cesse, son lot d'émotions tantôt plaisantes, tantôt déplaisantes. Les émotions intenses et déplaisantes peuvent générer du stress chez les futur.e.s enseignant.e.s, stress qui n'est pas toujours bien géré par certains d'entre eux.elles et qui peut mettre en péril la réussite de leurs stages (Montgomery, 2007 ; Champlain, 2008).

Si plusieurs situations sont source de stress pour certains stagiaires, ceux-ci ont généralement accès à des services psychologiques offerts dans leur établissement. Ces services mettent à la disposition des étudiant.e.s une panoplie de méthodes de psychothérapie visant à les aider à mieux gérer leur stress en situation de stage. Parmi les méthodes éprouvées, il existe les approches psychoéducatives (Young, Klosko et Weishaar, 2017), les techniques de relaxation et de respiration (Lupien, 2020), la pleine conscience (Stahl et Goldstein, 2013), la restructuration cognitive (Lecomte et Servant, 2020) ou le développement de l'intelligence émotionnelle ou des compétences émotionnelles (CE) (Gross, 2014) pour n'en nommer que quelques-unes. Depuis une vingtaine d'années, les CE font l'objet de multiples recherches auprès des personnes qui s'engagent dans une carrière professionnelle où les relations humaines sont essentielles. À titre d'exemple, des recherches menées auprès d'étudiant.e.s en médecine ont démontré que le développement des CE favorise la diminution du stress perçu lorsque ceux.celles-ci sont en résidence (Gorgas et al., 2015 ; Mintle, Greer, et Russo, 2019). Des résultats similaires ont été obtenus dans des recherches impliquant des étudiant.e.s en soins infirmiers (Dugé, Garncarzyk et Dosseville, 2018) et 


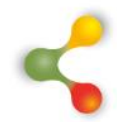

\section{REVUE HYBRIDE DE L'ÉDUCATION}

en éducation (Rey, Extremera et Pena, 2016; Vesely, Saklofsk et Nordstokke, 2014).

Bien que ces résultats soient encourageants, plusieurs écrits scientifiques mettent en évidence que la plupart de ces études comportent certaines faiblesses sur le plan théorique et méthodologique. L'absence de groupe témoin en est une. En effet, selon Kotsou et al. (2019), il serait difficile de comparer les résultats de certaines de ces recherches, car plusieurs d'entre elles n'ont pas de groupe témoin et n'utilisent pas les mêmes modèles théoriques relatifs aux CE. De plus, Sfetcu (2020) est d'avis que l'efficacité des programmes qui prétendent développer les CE est trop souvent remise en question par la communauté scientifique, ce qui appuie la pertinence de poursuivre les recherches empiriques sur le sujet (Kotsou et al., 2019).

Ainsi, la présente étude s'intéresse à la mise en place d'un programme qui vise le développement des CE chez les futur.e.s enseignant.e.s. Cet article tentera de mettre en évidence si un tel programme peut aider ces dernier.ère.s à mieux gérer leur CE et leur niveau de stress perçu en période de stage.

\section{Cadre théorique ou conceptuel}

\section{L'intelligence émotionnelle (IE) et les compétences émotionnelles} (CE)

Parmi les nombreux modèles portant sur l'intelligence émotionnelle (IE), deux d'entre eux favorisent le développement des CE en contexte professionnel (Sternberg, 2019). Le premier modèle (Salovey et Mayer, 1990) repose sur le traitement de l'information émotionnelle orienté sur des CE dites de base. II fut élaboré en tenant compte d'un ensemble de compétences liées au traitement et à la régulation des états émotionnels d'un individu (Salovey, Curaso et Mayer, 2016). Le second modèle, appelé modèle mixte et pour lequel il existe plusieurs conceptualisations (Bar-On, 1997 ; Goleman, 1995 ; Petrides et Furham, 2000), met l'accent sur les traits de personnalité. Par exemple, pour Goleman (1995), I'IE est une combinaison de traits de personnalité et d'habiletés émotionnelles qui sont acquises au fil des années. Son modèle est le plus répandu et a gagné beaucoup d'attention en dehors des cercles universitaires, notamment auprès des entreprises qui font la promotion des pratiques gagnantes en leadership (Sfetcu, 2020).

Bien que les modèles de l'IE se soient précisés au cours des années, il ne semble pas exister, à ce jour, de consensus dans la communauté scientifique quant à la définition du concept. Sternberg (2019) indique d'ailleurs que le sujet est encore récemment objet de controverses. En revanche, les deux grands modèles de l'IE ont permis aux experts de s'entendre sur une définition des CE (Mortiboys, 2005). Ainsi, les CE 


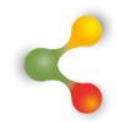

\section{REVUE HYBRIDE DE L'ÉDUCATION}

réfèrent aux différences dans la façon dont les personnes : 1) identifient, 2) expriment, 3) comprennent, 4) régulent, et 5) utilisent leurs émotions (volet intrapersonnel) et celles des autres (volet interpersonnel) (Mikolajczak, Quoidback, Kostou et Nelis, 2009). Ces cinq compétences se déclinent en trois niveaux, soit les connaissances qui déterminent les savoirs implicites et explicites de la personne sur ces cinq compétences, les habiletés qui se rapportent à la capacité de la personne à appliquer ses connaissances lors d'évènements chargés en émotion, et enfin, les dispositions qui sont les penchants de la personne à se comporter en général (Mikolajczak et al., 2009). De même, ces cinq compétences joueraient un rôle important sur le bien-être tant physique que psychologique, sur le niveau de stress, ainsi que sur la qualité des relations sociales des individus (Mikolajczak et al., 2015). Le modèle de Mikolajczak et al. (2009) a été opérationnalisé par Brasseur, Grégoire, Bourdu et Mikolajczak (2013) par le biais d'un questionnaire qui permet de dresser un profil des CE chez un individu. Cet outil semble être de plus en plus utilisé en recherche, notamment dans les études qui mettent en relation les CE et le stress chez les enseignant.e.s (Genoud, Kappeler et Gay, 2020).

\section{Le stress perçu}

Le premier modèle du stress impliquant les émotions de Selye (1956), considéré comme trop linéaire, a laissé la place au modèle transactionnel qui tient compte des particularités de l'individu en interaction avec son environnement (Stora, 2010). Lazarus et Folkman (1984), à l'origine de ce modèle, avancent que le stress découle, en grande partie, de la perception (stress perçu) qu'a l'individu de la relation qu'il entretient entre lui-même, les moyens dont il dispose et les obstacles de son milieu. Selon ces deux auteurs, l'évaluation cognitive d'une situation possiblement stressante par la personne comporte deux dimensions: primaire et secondaire. L'évaluation primaire a pour but d'estimer si la situation est stressante ou pas. Dans le cas où la situation est évaluée comme stressante, un processus de questionnement se déclenchera chez la personne. Ces questions sont généralement de deux ordres : 1) est-ce que je fais face à une situation qui implique une perte ou une menace ?; 2) S'agit-il plutôt d'une situation qui représente un défi ou des bénéfices ? L'évaluation d'une perte ou d'une menace provoque des émotions à valence négative tandis que l'évaluation d'un défi ou des bénéfices suscite des émotions à valence positive. Quant à l'évaluation secondaire, elle consiste à l'identification, par l'individu, de ce qu'il peut mettre en place pour remédier à la situation stressante; il s'agit du contrôle perçu. De plus, Lazarus et Folkman (1984) sont d'avis que le stress est indissociable des émotions, en ce sens qu'elles jouent un rôle important dans le choix des stratégies d'ajustement (coping) auxquelles l'individu a recours lorsqu'il fait face à un stresseur potentiel. Le choix de stratégies d'ajustement inadéquates à une situation stressante dénote souvent, notamment, une mauvaise régulation émotionnelle chez l'individu. Par ailleurs, il est faux de considérer que le stress est quelque chose de purement subjectif. En effet, 


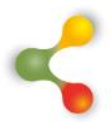

\section{REVUE HYBRIDE DE L'ÉDUCATION}

les évènements ont eux-mêmes des aspects qui les rendront sensibles d'être perçus comme stressants et il est important d'en être conscient si l'on désire moduler son niveau de stress (Lupien, 2020).

En résumé, dans le modèle transactionnel de Lazarus et Folkman (1984), le stress est envisagé comme le résultat de la transaction entre l'individu et son environnement. Ceci fait en sorte qu'il n'y a pas, a priori, de situation stressante. Tout évènement, qu'il s'agisse de petits soucis quotidiens ou d'une catastrophe majeure, peut être considéré stressant pour un individu. Ce n'est pas l'aspect objectivement stressant qui est déterminant, mais plutôt l'évaluation qu'en fait la personne en tenant compte de son histoire personnelle, de son soutien social, de ses ressources et de ses stratégies d'ajustement. Par conséquent, même une situation en apparence positive, telle qu'un mariage ou l'achat d'une première maison, peut se révéler très stressante pour certains.

À la lumière des définitions qui précèdent, il semble que les $C E$ pourraient avoir un impact positif sur le niveau de stress perçu des individus. Nelis et al. (2011) vont plus loin en affirmant qu'un haut niveau de $\mathrm{CE}$ constitue le facteur psychologique le plus prédictif à la résistance au stress. Forts de ce constat, Hansenne et al. (2014) ont développé, dans le cadre de leur recherche, un programme sur les CE qui s'inspire fortement du modèle des cinq CE de Mikolajczak et al. (2009). Les résultats de l'étude de Hansenne et al. (2014) démontrent qu'après avoir suivi leur programme, les participants du groupe expérimental ont augmenté significativement leurs capacités de régulation émotionnelle comparativement au groupe témoin. Puisque la régulation émotionnelle est un élément clé des $C E$, nous avons choisi d'examiner si le programme sur les CE de Hansenne et al. (2014) pourrait permettre à un groupe de futur.e.s enseignant.e.s d'améliorer leurs CE, et ainsi, diminuer leur stress perçu en période de stage.

\section{La présente étude}

Sur la base des résultats issus des recherches présentées précédemment, le but général de cette étude consiste à explorer si le programme sur les CE (Hansenne et al., 2014) pourrait contribuer à améliorer ces dernières chez les futur.e.s enseignant.e.s et à réduire leur stress perçu. Pour ce faire, trois hypothèses ont été formulées. Dans un premier temps, nous émettons l'hypothèse qu'entre le prétest et le posttest, les participants du groupe expérimental devraient avoir un meilleur profil de $\mathrm{CE}$ et une meilleure perception de leur stress. Au contraire, aucun changement sur le plan du profil de CE et du niveau de stress perçu ne devrait être observé chez les participants du groupe témoin entre ces deux temps de mesure. La deuxième hypothèse suggère qu'après avoir complété le programme sur les CE (Hansenne et al., 2014), les participants du groupe expérimental devraient présenter de meilleures CE comparativement au groupe témoin, et ce, tant sur le plan interpersonnel 


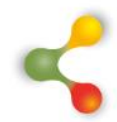

\section{REVUE HYBRIDE DE L'ÉDUCATION}

qu'intrapersonnel. Enfin, pour la dernière hypothèse, il est attendu qu'après leur participation au programme sur les CE (Hansenne et al., 2014), le niveau de stress perçu par le groupe expérimental devrait être moins élevé que celui du groupe témoin.

\section{Méthodologie}

\section{Participants et procédure}

L'échantillon constitué pour réaliser cette étude exploratoire comprend vingt étudiant.e.s inscrit.e.s dans un programme de formation à l'enseignement de l'Université de l'Alberta. Au moment de la collecte des données, tous les participant.e.s étaient en quatrième année de leur programme et étaient sur le point de débuter leur dernier stage avant l'obtention de leur diplôme. Les milieux de stages étaient comparables sur le plan socio-économique. Par contre, six des vingt participant.e.s ont indiqué que leur école manquait de ressources destinées à soutenir les élèves ayant des besoins éducatifs particuliers. Ces dernier.ère.s ont été réparti.e.s aléatoirement en deux groupes (le groupe témoin et le groupe expérimental), pour répondre aux exigences d'un devis expérimental, chaque groupe étant composé de dix participants dont cinq femmes et cinq hommes. L'âge moyen du groupe témoin est de 30 ans et 8 mois (écarttype : 5 ans et 4 mois) et celui du groupe expérimental est de 29 ans et 9 mois (écart-type : 6 ans et 2 mois). Les deux groupes sont considérés comme équivalents sur le plan de l'âge $(p=0,733)$. Enfin, dix des vingt participant.e.s étaient engagé.e.s dans un programme de Baccalauréat en Éducation après-diplôme $(\mathrm{BEd} / \mathrm{Ad})$ étant donné que ces derniers avaient déjà un diplôme universitaire de $1^{\text {er }}$ cycle.

Avant de débuter cette étude, toutes les procédures de réalisation ont été approuvées par le comité d'éthique de l'Université de l'Alberta. Tous les participant.e.s ont été informé.e.s de l'objectif et du déroulement de l'étude et ont signé un formulaire de consentement libre et éclairé.

Après avoir été répartis aléatoirement dans chaque groupe, les participant.e.s du groupe expérimental ont rempli un questionnaire sur leur niveau de CE ainsi qu'un questionnaire sur leur niveau de stress perçu juste avant le début de leur stage en milieu scolaire, puis pendant la deuxième semaine de leur stage. Les participant.e.s du groupe témoin ont également complété ces deux questionnaires, mais n'ont pas pris part au programme sur les CE.

\section{Programme sur les CE}

En raison de la pandémie de la COVID-19 et de la suspension des activités de formation en présentiel à l'Université de l'Alberta, dans le cadre de la présente étude, et ce, contrairement à la proposition originale de Hansenne et al. (2014), le programme a dû être offert entièrement en ligne. 


\section{$\&$}

\section{REVUE HYBRIDE DE L'ÉDUCATION}

Cependant, le contenu du programme n'a pas eu à être modifié. Ce programme comporte six modules répartis sur une période de six semaines, chaque module ayant une durée de trois heures, totalisant 18 heures de formation. Le fait que les modules soient répartis sur six semaines permet aux participant.e.s d'intégrer les apprentissages et de les mettre en pratique au quotidien. Les modules traitent essentiellement des cinq CE suggérées par Mikolajczak et al. (2009), soit l'identification, la compréhension, l'expression, la régulation et l'utilisation de ses propres émotions et de celles d'autrui. Tel que prévu par les auteur.rice.s, les contenus des modules ont été proposés grâce à différentes modalités pédagogiques : courtes présentations par l'auteur.e principal.e, clips vidéo, jeux de rôle, vignettes cliniques, forums de discussion, travaux en dyade et tenue de journaux de bord (voir Annexe 1 pour plus de détails sur les modules du programme).

\section{Instruments de mesure}

Le Profil des Compétences Émotionnelles de Brasseur et al. (2013) est un outil standardisé comptant 50 énoncés. 10 sous-échelles portant sur chacune des cinq composantes de base des CE (Mikolajczak et al., 2009), déclinée selon le volet intrapersonnel et le volet interpersonnel, peuvent être calculées (Tableau 1). Les participants répondent à chaque énoncé grâce à une échelle de Likert en 5 points $(1=$ totalement en désaccord et 5 = totalement en accord). Les énoncés sont formulés ainsi : "Je ne me base jamais sur mes émotions pour orienter ma vie» ou "Je peux facilement obtenir ce que je désire des autres ». Plus les scores aux souséchelles sont élevés, plus l'individu est considéré comme ayant de bonnes CE. Selon Brasseur et al. (2013), cet instrument possède une bonne cohérence interne $(\alpha=0.88)$.

Tableau 1 : Les 10 sous-échelles du Profil de Compétences Émotionnelles (Brasseur et al., 2013)

\begin{tabular}{lll}
\hline Sous-échelles & Soi & Autrui \\
\hline Identification & $\begin{array}{l}\text { Identifier (reconnaître) ses } \\
\text { émotions }\end{array}$ & $\begin{array}{l}\text { Identifier (reconnaître) les } \\
\text { émotions d'autrui }\end{array}$ \\
\hline Compréhension & Comprendre ses émotions & $\begin{array}{l}\text { Comprendre les émotions } \\
\text { d'autrui }\end{array}$ \\
\hline Expression & Exprimer ses émotions & $\begin{array}{l}\text { Étre à l'écoute des } \\
\text { émotions d'autrui }\end{array}$ \\
\hline Régulation & Réguler ses émotions & $\begin{array}{l}\text { Réguler les émotions } \\
\text { d'autrui }\end{array}$ \\
\hline Utilisation & Utiliser ses émotions & $\begin{array}{l}\text { Utiliser les émotions } \\
\text { d'autrui }\end{array}$ \\
\hline & & \\
\hline Numéro 1 & & \\
\hline
\end{tabular}




\section{$\&$}

\section{REVUE HYBRIDE DE L'ÉDUCATION}

Le Perceived Stress Scale est un questionnaire conçu par Cohen, Kamarck, et Mermelstein (1983). II s'agit d'un questionnaire qui permet d'évaluer l'importance avec laquelle des situations de la vie sont généralement appréhendées comme menaçantes par l'individu, c'est-àdire imprévisibles, incontrôlables et difficiles, et ce, depuis les 30 derniers jours (Langevin, Beni, François et Riou, 2015). II existe trois versions standardisées de cet outil, dont une en français. Cette dernière version a été retenue pour la présente étude et est composée de 10 énoncés. Les participant.e.s doivent répondre aux énoncés en choisissant une réponse de 1 à 5 sur une échelle Likert ( $1=$ jamais ; $5=$ très souvent). Les énoncés sont, par exemple : «Au cours du dernier mois, avez-vous été capable de maitriser votre énervement ? ». Le score total peut varier de 10 à 50 et est interprété de la façon suivante : lorsqu'il se situe en dessous de 21 , la personne est en mesure de gérer son stress ; entre 22 et 26 , la personne sait gérer son stress la plupart du temps même si elle rencontre quelques défis dans certaines situations; au-dessus de 27, la personne subit les situations stressantes sans savoir y faire face. Le questionnaire a été validé auprès de plusieurs échantillons et démontre des qualités psychométriques satisfaisantes. Des alphas variant de 0,84 à 0,86 pour plusieurs échantillons différents ont été répertoriés (Andreou et al., 2011; Cohen et al., 1983 ; Lee, 2012). Par ailleurs, cet outil se base sur le modèle de stress de Lazarus et Folkman (1984) impliquant une évaluation du caractère menaçant du stresseur et des capacités des personnes à y faire face. Enfin, l'échelle est adaptée au domaine professionnel, notamment pour les professionnel.le.s de l'enseignement (Langevin et al., 2015).

\section{Stratégies d'analyse}

Les réponses des participant.e.s aux deux questionnaires utilisés ont été considérées comme les variables dépendantes à l'étude. La taille de l'échantillon nous a conduits à mener des analyses statistiques non paramétriques. La comparaison des résultats de chaque groupe au prétest et au posttest (hypothèse 1), pour chaque outil de mesure, a été faite à partir de tests de Wilcoxon. Au contraire, des tests $U$ de Mann-Whitney pour échantillons indépendants ont été utilisés pour comparer les résultats des participant.e.s du groupe témoin et du groupe expérimental au posttest pour chaque outil de mesure (hypothèses 2 et 3 ).

\section{Résultats}

Avant de répondre aux hypothèses de recherche formulées précédemment, une vérification de l'équivalence des groupes au prétest sur le plan du profil des CE et de la perception de leur stress a été effectuée. Les résultats aux tests $U$ de Mann-Whitney indiquent que les deux groupes ne diffèrent pas avant que le programme sur les CE (Hansenne et al., 2014) soit mis en place. Les participant.e.s de deux 


\section{REVUE HYBRIDE DE L'ÉDUCATION}

groupes présentent donc un profil de CE et un niveau de stress perçu relativement similaire au prétest.

Pour répondre à la première hypothèse, les scores des deux groupes ont été comparés indépendamment entre le prétest et le posttest pour chaque outil de mesure. Ces analyses servent à mettre en évidence un éventuel changement à l'intérieur d'un même groupe entre les deux temps de mesure. Les scores obtenus et les résultats des analyses réalisées figurent dans le Tableau 2 pour le groupe témoin et dans le Tableau 3 pour le groupe expérimental. Les résultats des analyses indiquent que, pour le groupe témoin, seuls les scores globaux des CE sur les volets intrapersonnel et interpersonnel et le score de la sous-échelle d'utilisation des émotions d'autrui diffèrent entre le prétest et le posttest (CE intrapersonnelles : $p=0,02$; CE interpersonnelles : $p=0,01$; utilisation des émotions d'autrui : $p=0,02$ ). Ainsi, les scores des sous-échelles d'identification, de compréhension, d'expression, de régulation et d'utilisation des émotions tant personnelles qu'à l'égard d'autrui (sauf l'utilisation) ne changent pas significativement avant et après le programme d'intervention pour ce groupe. Notons toutefois que les scores d'identification des émotions personnelles et d'autrui et d'écoute des émotions d'autrui approchent le seuil de significativité (respectivement $p=0,06 ; p=0,06 ; p=0,08$ ). En outre, le niveau de stress perçu par les participants du groupe témoin s'avère être sensiblement le même entre le prétest et le posttest.

Tableau 2 : Moyennes (et écart-types) pour les sous-échelles du profil de CE et le niveau de stress perçu du groupe témoin au prétest et au posttest

\begin{tabular}{|c|c|c|c|c|}
\hline & \multirow{2}{*}{ Prétest } & \multirow{2}{*}{ Posttest } & \multicolumn{2}{|c|}{ Tests de Wilcoxon } \\
\hline & & & $\mathbf{z}$ & $\mathbf{p}$ \\
\hline $\begin{array}{l}\text { Identification des émotions } \\
\text { personnelles }\end{array}$ & $3,82(0,56)$ & $3,70(0,54)$ & $-1,897^{a}$ & $0,06^{\dagger}$ \\
\hline $\begin{array}{l}\text { Compréhension des } \\
\text { émotions personnelles }\end{array}$ & $3,30(0,60)$ & $3,26(0,62)$ & $-1,414^{a}$ & 0,16 \\
\hline $\begin{array}{l}\text { Expression des émotions } \\
\text { personnelles }\end{array}$ & $3,20(0,52)$ & $3,18(0,55)$ & $-0,447^{a}$ & 0,66 \\
\hline $\begin{array}{l}\text { Régulation des émotions } \\
\text { personnelles }\end{array}$ & $3,50(0,90)$ & $3,42(0,84)$ & $-1,265^{a}$ & 0,21 \\
\hline $\begin{array}{l}\text { Utilisation des émotions } \\
\text { personnelles }\end{array}$ & $3,36(0,57)$ & $3,30(0,55)$ & $-1,00^{a}$ & 0,32 \\
\hline CE intrapersonnelles & $3,43(0,42)$ & $3,37(042)$ & $-2,345^{a}$ & $0,02^{*}$ \\
\hline
\end{tabular}




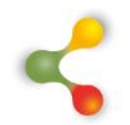

\section{REVUE HYBRIDE DE L’ÉDUCATION}

\begin{tabular}{|c|c|c|c|c|}
\hline $\begin{array}{l}\text { Identification des émotions } \\
\text { d'autrui }\end{array}$ & $3,68(0,52)$ & $3,58(0,54)$ & $-1,89^{a}$ & $0,06^{\dagger}$ \\
\hline $\begin{array}{l}\text { Compréhension des } \\
\text { émotions d'autrui }\end{array}$ & $3,56(0,72)$ & $3,50(0,77)$ & $-1,342^{a}$ & 0,18 \\
\hline $\begin{array}{l}\text { Écoute des émotions } \\
\text { d'autrui }\end{array}$ & $3,40(0,67)$ & $3,34(0,67)$ & $-1,732^{a}$ & $0,08^{\dagger}$ \\
\hline $\begin{array}{l}\text { Régulation des émotions } \\
\text { d'autrui }\end{array}$ & $3,42(0,85)$ & $3,28(0,89)$ & $-1,89^{a}$ & 0,59 \\
\hline $\begin{array}{l}\text { Utilisation des émotions } \\
\text { d'autrui }\end{array}$ & $3,20(0,52)$ & $3,02(0,38)$ & $-2,021^{a}$ & $0,02^{*}$ \\
\hline CE interpersonnelles & $3,45(0,55)$ & $3,34(0,56)$ & $-2,572^{a}$ & $0,01^{*}$ \\
\hline Stress perçu & $3,05(0,34)$ & $3,08(0,29)$ & $-0,624^{b}$ & 0,53 \\
\hline
\end{tabular}

En ce qui concerne le groupe expérimental (Tableau 3), on constate tout d'abord que plusieurs différences se révèlent significatives entre le prétest et le posttest. En cohérence, les analyses font ressortir un effet du temps de mesure pour toutes les sous-échelles qui portent sur les émotions personnelles des participants (identification: $p=0,04$; compréhension : $p=0,02$; expression : $p=0,03$; régulation : $p=0,03$ ) à l'exception de celle sur l'utilisation qui approche le seuil de significativité $(p=0,10)$, ainsi que pour le score global des CE intrapersonnelles $(p=0,005)$. Pour les sous-échelles des CE interpersonnelles, les résultats indiquent que des différences sont observées seulement pour la souséchelle de compréhension des émotions $(p=0,04)$ et le score global des CE interpersonnelles $(p=0,02)$. En d'autres termes, les participant.e.s de ce groupe semblent plus à même d'identifier, de comprendre, d'exprimer et de réguler leurs propres émotions et de comprendre celles des autres après avoir suivi le programme sur les CE (Hansenne et al., 2014) qu'au début de l'intervention. De plus, la perception de leur stress diminue au posttest $(p=0,005)$, ce qui tend à montrer un effet du programme sur la gestion du stress à l'intérieur de ce groupe. 


\section{REVUE HYBRIDE DE L'ÉDUCATION}

Tableau 3 : Moyennes (et écart-types) pour les sous-échelles du profil de CE et le niveau de stress perçu du groupe expérimental au prétest et au posttest

\begin{tabular}{|c|c|c|c|c|}
\hline & \multirow{2}{*}{ Pré test } & \multirow{2}{*}{ Post test } & \multicolumn{2}{|c|}{ Tests de Wilcoxon } \\
\hline & & & $\mathbf{z}$ & $\mathbf{p}$ \\
\hline $\begin{array}{l}\text { Identification des émotions } \\
\text { personnelles }\end{array}$ & $3,90(0,50)$ & $4,16(0,32)$ & $-2,041^{b}$ & $0,04^{*}$ \\
\hline $\begin{array}{l}\text { Compréhension des } \\
\text { émotions personnelles }\end{array}$ & $3,50(0,67)$ & $3,88(0,51)$ & $-2,392^{b}$ & $0,02^{*}$ \\
\hline $\begin{array}{l}\text { Expression des émotions } \\
\text { personnelles }\end{array}$ & $3,32(0,54)$ & $3,62(0,51)$ & $-2,214^{b}$ & $0,03^{*}$ \\
\hline $\begin{array}{l}\text { Régulation des émotions } \\
\text { personnelles }\end{array}$ & $3,26(0,83)$ & $3,58(0,67)$ & $-2,226^{b}$ & $0,03^{*}$ \\
\hline $\begin{array}{l}\text { Utilisation des émotions } \\
\text { personnelles }\end{array}$ & $3,28(0,84)$ & $3,36(0,87)$ & $-1,633^{b}$ & $0,10^{\dagger}$ \\
\hline CE intrapersonnelles & $3,45(0,51)$ & $3,72(0,45)$ & $-2,807^{b}$ & $0,005^{\star \star}$ \\
\hline $\begin{array}{l}\text { Identification des émotions } \\
\text { d'autrui }\end{array}$ & $3,68(0,53)$ & $3,72(0,42)$ & $-1,00^{b}$ & 0,32 \\
\hline $\begin{array}{l}\text { Compréhension des } \\
\text { émotions d'autrui }\end{array}$ & $3,32(0,66)$ & $3,46(0,62)$ & $-2,07^{b}$ & $0,04^{*}$ \\
\hline $\begin{array}{l}\text { Écoute des émotions } \\
\text { d'autrui }\end{array}$ & $3,54(0,67)$ & $3,58(0,68)$ & $-1,414^{b}$ & 0,16 \\
\hline $\begin{array}{l}\text { Régulation des émotions } \\
\text { d'autrui }\end{array}$ & $3,48(0,84)$ & $3,56(0,74)$ & $-1,342^{b}$ & 0,18 \\
\hline $\begin{array}{l}\text { Utilisation des émotions } \\
\text { d'autrui }\end{array}$ & $2,76(0,86)$ & $2,82(0,90)$ & $-1,342^{b}$ & 0,18 \\
\hline CE interpersonnelles & $3,35(0,56)$ & $3,43(0,50)$ & $-2,375^{b}$ & $0,02^{*}$ \\
\hline Stress perçu & $2,98(0,50)$ & $2,52(0,38)$ & $-2,825^{a}$ & $0,005^{\star \star}$ \\
\hline
\end{tabular}




\section{REVUE HYBRIDE DE L'ÉDUCATION}

Concernant les deux dernières hypothèses, ce sont seulement les moyennes (et écart-types) des deux groupes au posttest qui ont été utilisées pour réaliser les analyses requises. Les résultats sont récapitulés dans le Tableau 4 et mettent en évidence des différences significatives entre les deux groupes pour la sous-échelle de la compréhension des émotions personnelles $(p=0,03)$ et le niveau de stress perçu par les participants $(p=0,002)$. Ainsi, lorsque les deux groupes sont comparés à la fin du programme sur les CE (Hansenne et al., 2014), les participant.e.s du groupe expérimental présentent une meilleure compréhension de leurs propres CE et une meilleure perception de leur stress que les participant.e.s du groupe témoin. Toutefois, les scores des sous-échelles d'identification et d'expression des émotions personnelles et le score global des $C E$ intrapersonnelles démontrent une tendance (respectivement $p=0,06 ; p=0,06 ; p=0,07$.

\section{Tableau 4 : Moyennes (et écart-types) pour les sous-échelles du profil de CE et le niveau de stress perçu de chaque groupe au posttest}

\begin{tabular}{|c|c|c|c|c|}
\hline & \multirow{2}{*}{ Groupe témoin } & \multirow{2}{*}{$\begin{array}{l}\text { Groupe } \\
\text { expérimental }\end{array}$} & \multicolumn{2}{|c|}{$\begin{array}{l}\text { Tests U de Mann- } \\
\text { Whitney }\end{array}$} \\
\hline & & & $\mathbf{z}$ & $\mathbf{p}$ \\
\hline $\begin{array}{l}\text { Identification des émotions } \\
\text { personnelles }\end{array}$ & $3,70(0,54)$ & $4,16(0,32)$ & 1,914 & $0,06^{\dagger}$ \\
\hline $\begin{array}{l}\text { Compréhension des } \\
\text { émotions personnelles }\end{array}$ & $3,26(0,62)$ & $3,88(0,51)$ & 2,164 & $0,03^{*}$ \\
\hline $\begin{array}{l}\text { Expression des émotions } \\
\text { personnelles }\end{array}$ & $3,18(0,55)$ & $3,62(0,51)$ & 1,911 & $0,06^{\dagger}$ \\
\hline $\begin{array}{l}\text { Régulation des émotions } \\
\text { personnelles }\end{array}$ & $3,42(0,84)$ & $3,58(0,67)$ & 0,265 & 0,8 \\
\hline $\begin{array}{l}\text { Utilisation des émotions } \\
\text { personnelles }\end{array}$ & $3,30(0,55)$ & $3,36(0,87)$ & 0,114 & 0,91 \\
\hline CE intrapersonnelles & $3,37(042)$ & $3,72(0,45)$ & 1,780 & $0,07^{\dagger}$ \\
\hline $\begin{array}{l}\text { Identification des émotions } \\
\text { d'autrui }\end{array}$ & $3,58(0,54)$ & $3,72(0,42)$ & 0,573 & 0,58 \\
\hline $\begin{array}{l}\text { Compréhension des } \\
\text { émotions d'autrui }\end{array}$ & $3,50(0,77)$ & $3,46(0,62)$ & $-0,305$ & 0,8 \\
\hline
\end{tabular}




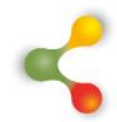

REVUE HYBRIDE DE L'ÉDUCATION

\begin{tabular}{lllll}
$\begin{array}{l}\text { Écoute des émotions } \\
\text { d'autrui }\end{array}$ & $3,34(0,67)$ & $3,58(0,68)$ & 0,841 & 0,44 \\
\hline $\begin{array}{l}\text { Régulation des émotions } \\
\text { d'autrui }\end{array}$ & $3,28(0,89)$ & $3,56(0,74)$ & 1,146 & 0,28 \\
\hline $\begin{array}{l}\text { Utilisation des émotions } \\
\text { d'autrui }\end{array}$ & $3,02(0,38)$ & $2,82(0,90)$ & $-0,459$ & 0,68 \\
\hline CE interpersonnelles & $3,34(0,56)$ & $3,43(0,50)$ & 0,597 & 0,63 \\
\hline Stress perçu & $3,08(0,29)$ & $2,52(0,38)$ & $-2,999$ & $0,002^{* *}$ \\
\hline${ }^{* *} p<0,01 ;{ }^{*} p<0,05 ;{ }^{\dagger} p<.10$ & & & \\
\hline
\end{tabular}

\section{Discussion}

L'objectif de cette étude exploratoire consistait à examiner si le programme sur les CE (Hansenne et al., 2014) pouvait contribuer à les améliorer chez de futur.e.s enseignant.e.s, et ainsi, diminuer leur stress perçu. Concernant la première hypothèse, les résultats démontrent qu'elle est partiellement confirmée. En effet, les scores obtenus par les participant.e.s du groupe expérimental se sont statistiquement améliorés entre le prétest et le posttest, ce qui va dans le sens de l'hypothèse formulée. Cependant, cette amélioration n'a pas été observée de façon unanime pour toutes les échelles du questionnaire du profil des $C E$, ce qui nécessite une certaine prudence dans l'interprétation des résultats. Par ailleurs, les résultats des participant.e.s du groupe témoin viennent infirmer, en partie, l'hypothèse énoncée, puisque ces derniers voient trois de leurs scores s'améliorer entre le prétest et le posttest. Ces résultats peuvent paraître surprenants et pour le moins inusités puisque, contrairement au groupe expérimental, les participant.e.s de ce groupe témoin n'ont bénéficié d'aucune intervention spécifique pour l'amélioration de leurs $C E$. Cette divergence pourrait s'expliquer par un effet de contagion au sein des deux groupes. En effet, on se souviendra que les participant.e.s ont été réparti.e.s de façon aléatoire dans le groupe expérimental et le groupe témoin et que tou.te.s les participant.e.s étaient en quatrième année et suivaient le même cursus universitaire. Cette situation a possiblement donné naissance à des discussions autour de cette recherche pouvant alors influencer ainsi la nature des réponses du groupe témoin au moment de la seconde passation du questionnaire sur le profil de CE. En revanche, toujours relativement à cette première hypothèse, les résultats obtenus au questionnaire de stress perçu n'ont pas changé entre le prétest et le posttest à l'intérieur du groupe témoin alors que c'est le cas pour le groupe expérimental, ce qui va dans le sens de l'hypothèse. 


\section{$\propto$ \\ REVUE HYBRIDE DE L'ÉDUCATION}

En ce qui a trait à la deuxième hypothèse, les résultats indiquent qu'elle est également en partie confirmée. Ainsi, à la fin du programme sur les CE (Hansenne et al., 2014), les participants du groupe expérimental présentent une meilleure compréhension de leurs propres CE que les participants du groupe témoin. En outre, les scores aux sous-échelles d'identification et expression des émotions personnelles démontrent une tendance. Toutefois, les résultats ne diffèrent pas significativement pour quatre des cinq sous-échelles des CE interpersonnelles, soit l'identification, l'écoute, la régulation et l'utilisation des émotions d'autrui. Tel que mis en évidence par Nozaki et Mikolajczak (2020), cette divergence peut s'expliquer, en partie, par le fait qu'il est plus difficile d'exercer une influence sur les émotions d'autrui que sur ses propres émotions, notamment sur la régulation des émotions d'autrui, surtout lorsque le programme d'intervention est intensif et se déroule sur une courte période de temps, comme ce fut le cas dans cette étude.

Enfin, concernant le niveau de stress perçu après la mise en place du programme sur les CE (Hansenne et al., 2014), les résultats du groupe expérimental sont significativement moins élevés que ceux du groupe témoin, ce qui vient confirmer la troisième hypothèse. Ces résultats sont cohérents avec ceux de la première hypothèse, qui ont fait ressortir une baisse du stress perçu par les participant.e.s du groupe expérimental entre les deux temps de mesure alors que ce n'était pas le cas pour ceux du groupe témoin. De plus, ces résultats sont en accord avec ceux de l'étude de Vesely et al. (2014) qui suggèrent qu'après avoir réalisé un programme d'intervention portant sur les CE, le groupe expérimental, composé de 23 futur.e.s enseignant.e.s, a vu son niveau de stress et d'anxiété diminuer significativement comparativement aux 26 futur.e.s enseignant.e.s du groupe témoin.

\section{Limites de l'étude et pistes de recherches futures}

Les principales limites de cette étude exploratoire reposent sur des considérations méthodologiques. Tout d'abord, cette étude quantitative aurait pu être accompagnée de l'analyse de données qualitatives issues des verbalisations des participant.e.s. Un devis de recherche mixte, dans ce cas, aurait non seulement enrichit la méthodologie, mais aurait également permis de recueillir de l'information plus détaillée sur la façon dont les participant.e.s ont amélioré ou non leurs CE après avoir suivi le programme proposé (Hansenne et al., 2014). Sachant que certaines écoles avaient moins de ressources que d'autres pour soutenir les élèves ayant des besoins éducatifs particuliers, par exemple, de l'information sur le contexte scolaire dans lequel les participant.e.s ont évolué durant leur stage aurait nuancé les résultats. De même, il serait également intéressant, dans des recherches ultérieures, d'inclure une mesure de stress physiologique tel qu'un test de cortisol salivaire ou un test de cortisol capillaire parmi les outils d'évaluation. Une telle mesure viendrait éliminer les facteurs de suggestibilité et de désirabilité sociale qu'engendrent des 


\section{$\&$}

\section{REVUE HYBRIDE DE L'ÉDUCATION}

données autorapportées sur le stress psychologique perçu. Une autre limite de cette étude se rapporte à la petite taille de notre l'échantillon. Le faible nombre de participant.e.s a pour effet de réduire le caractère généralisable des résultats de la présente étude. Cet aspect a été relevé comme une critique dans la méta-analyse de Kostsou et al. (2019) car la majorité des recherches répertoriées sont constituées de petits échantillons. Les recherches futures dans le domaine devront faire appel à des échantillons beaucoup plus grands afin de confirmer ou infirmer les tendances observées dans la présente étude. Finalement, nous n'avons pas effectué de suivi quelques mois après le posttest afin de s'assurer que les effets du programme sur les CE de Hansenne et al. (2014) se maintiennent dans le temps. Dans ces conditions, il est impossible de prévoir si les retombées de cette étude persistent à moyen et à long terme.

\section{Conclusion}

Malgré les limites énoncées précédemment, nous conclurons sur le fait que cette étude a permis de mettre en évidence des résultats prometteurs en termes d'amélioration des CE qui, par ricochet, peuvent affecter à la baisse le niveau de stress perçu par les futur.e.s enseignant.e.s durant leur stage. Ces résultats sont d'autant plus intéressants si l'on tient compte du fait que le programme sur les CE (Hansenne et al., 2014) mis en place dans la présente étude se donne habituellement en face à face. Dans le contexte actuel, il a dû être adapté pour une version entièrement en ligne à cause du confinement exigé par les autorités sanitaires. Cette version du programme démontre toute sa pertinence et son réalisme dans un contexte où les activités virtuelles deviennent de plus en plus fréquentes. Enfin, cette étude vient appuyer les recommandations de chercheurs à l'effet que les futur.e.s enseignant.e.s doivent développer leurs CE dès la formation initiale afin d'être mieux préparés à faire face aux défis qui les attendent durant leur carrière. En effet, puisque celle-ci entraîne souvent des émotions intenses qui sont sources de stress, en particulier durant les cinq premières années, le bienêtre psychologique et physique des enseignant.e.s débutant.e.s s'en trouve malheureusement affecté de façon négative, ce qui nécessite d'être pris au sérieux par les universités qui les forment (Beaumont et Garcia, 2020 ; Lafortune, Saint-Pierre et Martin 2005 ; Lafranchise, 2010 ; Pelletier, 2015).

\section{Remerciements}

Nous tenons à remercier Alberta Education pour la subvention accordée en soutien à cette recherche. 


\section{REVUE HYBRIDE DE L'ÉDUCATION}

\section{Références}

Andreou, E., Alexopoulos, E.C., Lionis, C., Varvogli, L., Gnardellis, C., Chrousos, G.P. et Darviri, C. (2011). Perceived Stress Scale: reliability and validity study in Greece. International Journal of Environmental Research and Public Health, 8(8), 3287-3298. https://doi.org/10.3390/ijerph8083287

Bar-On, R. (1997). The emotional quotient inventory (EQ-if): A test of emotional intelligence. Multi-Health Systems.

Beaumont, C. et Garcia, N. (2020). L'apprentissage socioémotionnel à l'école primaire: compétences attendues des enseignants et formation initiale. Recherches en éducation, 41, 60-73. https://doi.org/10.4000/ree.544

Bonello, M., Landry, G., Panaccio, A. et Chadwick, I. (2019). Facteurs de stress chez des stagiaires de gestion. Humain et organisation, 5(2), $12-22$.

Brasseur, S., Grégoire, J., Bourdu, R. et Mikolajczak, M. (2013). The Profile of Emotional Competence (PEC): Development and Validation of a Self-Reported Measure that Fits Dimensions of Emotional Competence Theory. PLOS ONE, 8(5). https://doi.org/10.1371/journal.pone.0062635

Chaplain, R.P. (2008). Stress and psychological distress among trainee secondary teachers in England. Educational Psychology, 28(2), 195-209. https://doi.org/10.1080/01443410701491858

Cohen, S., Kamarck, T. et Mermelstein, R. (1983). A global measure of perceived stress. Journal of Health and Social Behavior, 24(4), 385-396. https://doi.org/10.2307/2136404

Desbiens, J.-F., Borges, C. et Spallanzani, C. (2012). J'ai mal à mon stage. Problèmes et enjeux de la formation pratique en enseignement. Presses de l'Université du Québec.

Dugué, M, Garncarzyk, C. et Dosseville, F. (2018). Déterminants psychologiques du stress chez les étudiants en soins infirmiers. Revue d'Épidémiologie et de Santé Publique, 66(6), 347-354. https://doi.org/10.1016/j.respe.2018.09.004

Genoud, P.A., Kappeler, G. et Gay, P. (2020). Faut-il former les enseignants afin qu'ils cherchent à diminuer les émotions négatives de leurs élèves ou qu'ils leur apprennent à renforcer leurs émotions positives ?. Recherches en éducation, 41, 31-45. https://doi.org/10.4000/ree.519 


\section{REVUE HYBRIDE DE L'ÉDUCATION}

Goleman, D. (1995). Emotional intelligence. Bantam Books Inc.

Gorgas, D.L., Greenberger, S., Bahner, D.P. et Way, D.P. (2015). Teaching emotional intelligence: a control group study of a brief educational intervention for emergency medicine residents. Western Journal of Emergency Medicine, 16(6), 899-906. https://doi.org/10.5811/westjem.2015.8.27304

Goyette, N., Martineau, S., Gagnon, B. et Bazinet, J. (2020). Les effets d'une approche pédagogique préconisant la psychopédagogie du bienêtre sur la réussite éducative d'élèves du primaire. Revue hybride de l'éducation, 4(4), 1-23. https://doi.org/10.1522/rhe.v4i4.1072

Gross, J.J. (2014). Handbook of Emotion Regulation. Guilford.

Hansenne, M., Nélis, D., Feyers, D., Salmon, E. et Majerus, S. (2014). Better Neuronal Efficiency After Emotional Competences Training: An fMRI Study. Psychologica Belgica, 54(4), 328-349. http://doi.org/10.5334/pb.av

Kotsou, I., Mikolajczak, M., Heeren, A., Grégoire, J. et Leys, C. (2019). Improving emotional intelligence. A systematic review of existing work and future challenges. Emotion Review, 11(2), 151-165. https://doi.org/10.1177/1754073917735902

Lafortune, L., Saint-Pierre, L. et Martin, D. (2005). Compétence émotionnelle dans l'accompagnement. Dans L. Lafortune, M.-F. Daniel, P.-A. Doudin, F. Pons et O. Albanese (dir.), Pédagogie et psychologie des émotions: vers la compétence émotionnelle (p.87-118). Presses de l'Université du Québec.

Lafranchise, N. (2010). Analyse du cheminement de personnes enseignante au plan de la compétence émotionnelle et de sa prise en compte, dans le contexte de l'insertion professionnelle et d'une démarche d'accompagnement dans une perspective socioconstructiviste [thèse de doctorat inédite, Université du Québec à Trois-Rivières].

Langevin, V., Beni, S., François, M. et Riou, A. (2015). Échelle de stress perçu. Références en santé au travail, 143, 101-104.

Lazarus, R.S. et Folkman, S. (1984). Stress, appraisal and coping. Springer.

Lecomte, C. et Servant, D. (2020). Les thérapies comportementales, cognitives et émotionnelles en 150 fiches. Elsevier Masson. 


\section{REVUE HYBRIDE DE L'ÉDUCATION}

Lee, E.-H. (2012). Review of the Psychometric Evidence of the Perceived Stress Scale. Asian Nursing Research, 6(4), 121-127.

Lupien, S. (2020). Par amour du stress. Éditions Va savoir.

Mikolajczak, M., Avalosse, H., Vancorenland, S., Verniest, R., Callens, M., van Broeck, N., Fantini-Hauwel, C. et Mierop, A. (2015). A nationally representative study of emotional competence and health. Emotion, 15(5), 653-667. https://doi.org/10.1037/emo0000034

Mikolajczak, M., Quoidback, J., Kostou, I. et Nelis, D. (2009). Les compétences émotionnelles. Dunod.

Mintle, L.S., Greer, C.F. et Russo, L.E. (2019). Longitudinal Assessment of Medical Student Emotional Intelligence Over Preclinical Training. Journal of the American Osteopathic Association, 119(4), 236-242. https://doi.org/10.7556/jaoa.2019.039

Montgomery, C. (2007). Stress et résolution de problèmes sociaux : deux contextes d'enseignement francophones. Nouveaux cahiers de la recherche en éducation, 10(2), 111-129. https://doi.org/10.7202/1018166ar

Mortiboys, A. (2005). Teaching with emotional intelligence. Routledge.

Nelis, D., Kotsou, I., Quoidbach, J., Hansenne, M., Weytens, F., Dupuis, P. et Mikolajczak, M. (2011). Increasing emotional competence improves psychological and physical well-being, social relationships, and employability. Emotion, 11(2), 354-366. https://doi.org/10.1037/a0021554

Nozaki, Y. et Mikolajczak, M. (2020). Extrinsic emotion regulation. Emotion, 20(1), 10-15. https://doi.org/10.1037/emo0000636

Pelletier, M.-A. (2015). La gestion des émotions face aux situations stressantes à l'école: les finissants stagiaires en éducation préscolaire et enseignement primaire se sentent-ils prêts?. Éducation et francophonie, 43(2), 201-218. https://doi.org/10.7202/1034492ar

Petrides, K. V. et Furnham, A. (2000). On the dimensional structure of emotional intelligence. Personality and Individual Differences, 29(2), 313-320. https://doi.org/10.1016/S0191-8869(99)00195-6

Rey, L., Extremera, N. et Pena. M. (2016). Emotional competence relating to perceived stress and burnout in Spanish teachers: a mediator model. PeerJ. https://doi.org/10.7717/peerj.2087 


\section{REVUE HYBRIDE DE L'ÉDUCATION}

Salovey, P. et Mayer, J.D. (1990). Emotional Intelligence. Imagination, Cognition and Personality, 9(3), 185-211. https://doi.org/10.2190/DUGG-P24E-52WK-6CDG

Salovey, P., Caruso, D.R. et Mayer, J.D. (2016). The ability model of emotional intelligence: Principles and updates. Emotion Review, 8(4), 290-300. https://doi.org/10.1177/1754073916639667

Selye, H. (1956). The stress and distress. Mc Graw-Hill.

Sfetcu, N. (2020). Émotions et intelligence dans les organisations. MultiMedia Publishing.

Stahl, B. et Goldstein, E. (2013). Apprendre à méditer : La méthode MBR à la portée de tous. Éditions des Arènes.

Sternberg, R.J. (2019). Human Intelligence. An Introduction. Cambridge University Press.

Stora, J.B. (2010). Le stress. Que sais-je?

Vandercleyen, F., L'Hostie, M. et Dumoulin, M.-J. (2019). Le groupe de codéveloppement professionnel pour former à l'accompagnement des stagiaires. Conditions, enjeux et perspectives. Presses de l'Université du Québec.

Vesely, A.K., Saklofske, D.H. et Nordstokke, D.W. (2014). El training and pre-service teacher wellbeing. Personality and Individual Differences, 65, 81-85. https://doi.org/10.1016/j.paid.2014.01.052

Weber, J.M. (2015). L'impact de la relation tuteur-stagiaire sur la formation des enseignants. Évaluer : Journal international de recherche en éducation et formation, 3, 29-42. http://hdl.handle.net/10993/32363

Young, J.E., Klosko, J.S. et Weishaar, E. (2017). La thérapie des schémas. De Boeck Supérieur. 


\section{REVUE HYBRIDE DE L'ÉDUCATION}

\section{Annexe 1 : Les cinq modules du programme sur les CE de Hansenne}

et al. (2014)

Module 1 : Comprendre ses émotions et celles d'autrui

- Prétest

- Présentation du programme sur les CE et explication des concepts clés

- Vidéoclip sur l'importance des émotions dans notre vie

- Forum de discussion

- Devoir 1 (vignette clinique A)

- Durée 3 heures

Module 2 : Identifier ses émotions et celles d'autrui

- Retour sur le module 1

- Identification des émotions en utilisant la technique des trois portes

- Identification des émotions en situation de communication non verbale

- Forum de discussion

- Devoir 2 (vignette clinique B)

- Durée 3 heures

Module 3 : Expression de ses propres émotions et écoute des émotions d'autrui

- Retour sur le module 2

- Mini-session sur la communication de base

- Jeux de rôle suivi d'écoute empathique

- Travail en dyade en chambre zoom 


\section{REVUE HYBRIDE DE L'ÉDUCATION}

- Forum de discussion

- Devoir 3 (vignette clinique C)

- Durée 3 heures

Module 4 : La régulation de ses propres émotions et celles d'autrui

- Retour dur le module 3

- Technique de relaxation

- Réévaluation cognitive positive

- Travail en petit groupe en chambre zoom

- Devoir 4 (vignette clinique D)

- Durée 3 heures

Module 5 : L'utilisation de ses émotions et celles d'autrui

- Retour sur le module 4

- Vidéoclip sur l'importance de l'approche positive des émotions

- Exercice de gratitude en chambre zoom

- Forum de discussion

- Devoir 5 (vignette clinique E)

- Durée 3 heures

Module 6 : L'importance des émotions

- Retour sur le module 5

- L'importance des émotions positives

- Discussions en petit groupe en chambre zoom

- Recourir aux émotions positives afin de promouvoir de bons sentiments 


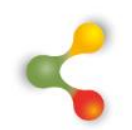

REVUE HYBRIDE DE L'ÉDUCATION

- Retour sur l'ensemble des modules

- Durée 3 heures 